\title{
Outburst evolution, historic light curve and a flash-ionized nebula around the WZ Sge-type object PNV J03093063+2638031 ^
}

\author{
U. Munari $^{1}$, R. Jurdana-Šepić ${ }^{2}$, P. Ochner ${ }^{1}$, and G. Cherini ${ }^{3}$
}

${ }^{1}$ INAF Osservatorio Astronomico di Padova, 36012 Asiago (VI), Italy e-mail: ulisse.munari@oapd.inaf.it

2 Physics Department, University of Rijeka, Radmile Matejčić 2, 51000 Rijeka, Croatia

3 ANS Collaboration, c/o Osservatorio Astronomico, via dell'Osservatorio 8, 36012 Asiago (VI), Italy

Received 19 June 2015 / Accepted 7 September 2015

\begin{abstract}
We have monitored the 2014 superoutburst of the WZ Sge-type transient PNV J03093063+2638031 for more than four months, from $V=11.0$ maximum brightness down to $V=18.4 \mathrm{mag}$, close to quiescence value, by obtaining $B V R_{\mathrm{C}} I_{\mathrm{C}}$ photometry and low resolution fluxed spectroscopy. The evolution was normal and no late-time "echo" outbursts were observed. The absolute integrated flux of emission lines kept declining along the superoutburst, and their increasing contrast with the underlying continuum was simply the result of the faster decline of the continuum compared to the emission lines. Inspection of historical Harvard plates covering the 1899-1981 period did not reveal previous outbursts, neither "normal" nor "super". We discovered an extended emission nebula (radius 1 arcmin) around PNV J03093063+2638031, that became visible for a few months as the result of photo-ionization from the superoutburst of the central star. The nebula is not present on Palomar I and II sky survey images and it quickly disappeared when the outburst was over. From the rate at wich the ionization front swept through the nebula, we derive a distance of $\sim 120 \mathrm{pc}$ to the system. The nebula is density bounded with an outer radius of $0.03 \mathrm{pc}$, and the absolute magnitude of the central star in quiescence is $M_{V} \sim 14.2 \mathrm{mag}$. The electron density in the nebula is estimated to be $10^{5} \mathrm{~cm}^{-3}$ from the observed recombination time scale. Given the considerable substructures seen across the nebula, a low filling factor is inferred. Similar nebulae have not been reported for other WZ Sge objects and the challenges posed to models are considered.
\end{abstract}

Key words. novae, cataclysmic variables

\section{Introduction}

According to the reports that appeared on Transient Object Confirmation Page Reports (TOCP) web page of the IAU Central Bureau for Astronomical Telegrams ${ }^{1}$, PNV J03093063+2638031 was discovered on 2014 Oct. 29.630 UT (JD $=2456960.130$ ) as a $\sim 11.0$ mag optical transient by S. Ueda observing from Japan with a $25 \mathrm{~cm}$ reflector. The astrometric position was later refined by $\mathrm{S}$. Kiyota to have end figures 29.86 and $044^{\prime \prime} 49$ in RA and Dec, 29.77 and $04 . .3$ by T. Noguchi, and 29.77 and $04 . ' 6$ by M. Caimmi, respectively, (cf. TOCP). We take the straight average of these three determinations as the best available J2000 astrometric position of the transient: $\alpha=03^{\mathrm{h}} 09^{\mathrm{m}} 29^{\mathrm{s}} .80( \pm 0.03)$ $\delta=+26^{\circ} 38^{\prime} 04^{\prime \prime} 5\left( \pm 0^{\prime}{ }^{\prime} 1\right)$ which is 12.5 arcsec away from the originally reported position and suggests OT J030929.8+263804 as a possibly more appropriate name for the transient.

P. Berardi (cf. TOCP) on Oct. 30.8 UT and Santangelo \& Gambogi (2014) also on Oct. 30.8 UT obtained low-resolution spectroscopic observations of PNV J03093063+2638031, showing a blue continuum, narrow $\mathrm{H}$-alpha line profile and broad absorptions for the other members of Balmer lines, which suggested the object is a cataclysmic variable (CV) in outburst. Not much else has been published on this transient. Alert-news

\footnotetext{
* Spectra of Fig. 2 and full Table 4 are only available at the CDS via anonymous ftp to cdsarc.u-strasbg. fr $(130.79 .128 .5)$ or via http://cdsarc.u-strasbg.fr/viz-bin/qcat?]/A+A/584/A12 1 http://www. cbat. eps.harvard. edu/unconf/tocp . xml
}

issued by VSNET $^{2}$ reported on the detection of early superhumps in time resolved photometry of PNV J03093063+2638031, with an amplitude of 0.025 mag and a period of 0.05613 days $\left(=80^{\mathrm{m}} 49^{\mathrm{s}}\right)$ on Oct. 31/Nov. 1 . The superhumps remained visible during following weeks.

The transient was soon classified as a new WZ Sge-type object on these VSNET reports. This kind of classification is sound, given the presence of early superhumps, the large amplitude of the recorded outburst, and its rarity (no previous outbursts recorded). WZ Sge stars are an extreme case of SU UMatype CVs, with intervals between superoutbursts lasting decades, while normal outbursts are few and far in between (Hellier 2001). WZ Sge itself undergoes a superoutburst every $\sim 30 \mathrm{yr}$ (events recorded in 1913, 1946, 1978 and 2001; Kuulker et al. 2011) and has never been seen to undergo a normal outburst in between. At the opposite end of SU UMa-type of CVs are the ER UMa stars, which spend typically a third to half of their time in superoutbursts, going into one every one to two months. Outside superoutbursts, the ER UMa stars experience a rapid series of normal outbursts, showing one every few days.

In this paper we report about (a) the photometric and spectroscopic evolution of PNV J03093063+2638031 (hereafter PNV for short) during the 2014 superoutburst from our $B V R_{\mathrm{C}} I_{\mathrm{C}}$ photometry and fluxed spectroscopy; (b) the results of our inspection of the Harvard plate stack to reconstruct the past photometric

2 http://www.kusastro.kyoto-u.ac.jp/vsnet/ 


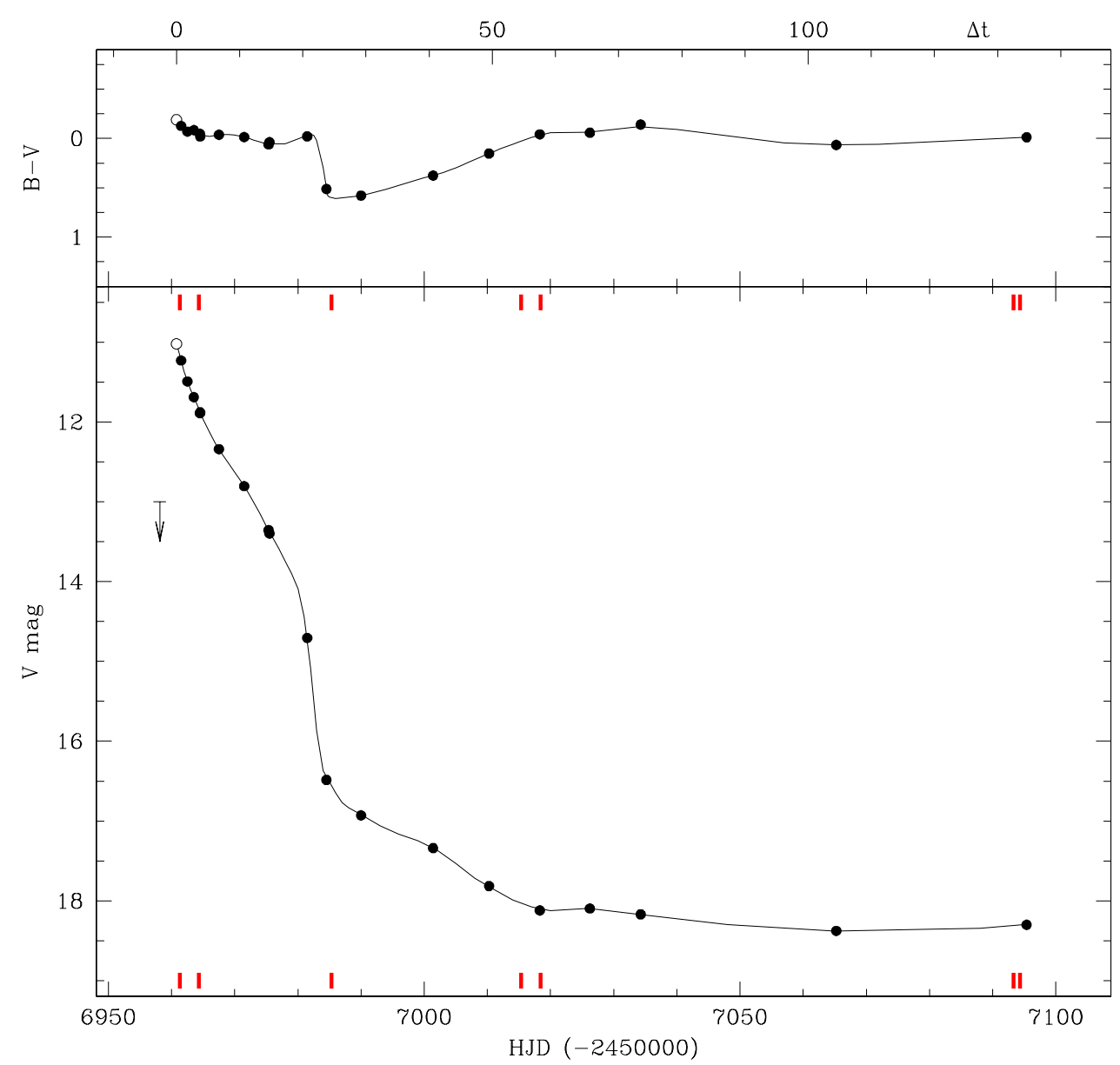

Fig. 1. Photometric evolution of PNV J03093063+2638031 during the 2014 superoutburst, from our observations in Table 1. The thick markers point to the epochs of the spectra listed in Table 2. Time on the upper abscissae is counted from the epoch of maximum brightness on 2014 Oct. 30.28 UT (JD = 2456 960.78). For the open circles and the arrow, see text (Sect. 5).

history of the object; and (c) the discovery of a spatially resolved nebula around PNV, flash-ionized by the 2014 superoutburst.

\section{Observations}

The photometric observations of PNV during the 2014 superoutburst were carried out with three different telescopes. ANS Collaboration telescope N. 11, located in Trieste (Italy), was used when the object was bright. It is a $13 \mathrm{~cm} \mathrm{f} / 6.6$ Vixen ED130SS refractor equipped with Custom Scientific $U B V R_{\mathrm{C}} I_{\mathrm{C}}$ photometric filters and a SBIG ST10XME CCD camera, $2184 \times$ 1472 array, $6.8 \mu \mathrm{m}$ pixel providing a focal plane scale of $1.63^{\prime \prime} /$ pix and a field of view of $60^{\prime} \times 40^{\prime}$. Technical details and operational procedures of the ANS Collaboration network of telescopes running since 2005, are presented by Munari et al. (2012). Detailed analysis of the photometric performances and measurements of the actual transmission profiles for all the photometric filter sets in use at all telescopes is presented by Munari \& Moretti (2012). During the latest phases of the outburst, larger instruments were used to measure PNV, namely the Asiago 67/92 cm Schmidt and $1.82 \mathrm{~m}$ reflector telescopes. The $67 / 92 \mathrm{~cm} \mathrm{f} / 3.2 \mathrm{Schmidt}$ telescope is equipped with Custom Scientific $B V R_{\mathrm{C}} I_{\mathrm{C}}$ photometric filters and a SBIG STL-11000MC2 CCD camera, $4008 \times 2672$ array, $9 \mu \mathrm{m}$ pixel providing a focal plane scale of $0.86^{\prime \prime} /$ pix and a field of view of $58^{\prime} \times 38^{\prime}$. The $1.82 \mathrm{~m}$ telescope was used with the AFOSC spectrograph-imager, which houses $U B V R_{\mathrm{C}} I_{\mathrm{C}}$ filters and an Andor DW436-BV CCD camera, $2048 \times 2048$ array, $13.5 \mu \mathrm{m}$ pixel providing a focal plane scale of $0.26^{\prime \prime} /$ pix and a field of view of $8.9^{\prime} \times 8.9^{\prime}$. All measurements on PNV were performed with aperture photometry (the sparse field not requiring the use of PSF fitting) against a photometric sequence located around the variable. The sequence was calibrated from APASS survey data (Henden et al. 2012; Henden \& Munari 2014) using the transformation equation calibrated in Munari et al. (2014). Our measurements are plotted in Fig. 1 and presented in Table 1, where the reported uncertainties are total error budgets, combining quadratically the error on the variable with the uncertainty of the transformation from the istantaneous local photometric system to the standard one as defined by the local photometric sequence.

Low-resolution spectroscopy of PNV was obtained with the $1.22 \mathrm{~m}$ telescope $+\mathrm{B} \& \mathrm{C}$ spectrograph operated in Asiago by the Department of Physics and Astronomy of the University of Padova. The CCD camera is a ANDOR iDus DU440A with a back-illuminated E2V 42-10 sensor, $2048 \times 512$ array of $13.5 \mu \mathrm{m}$ pixels. It is highly efficient in the blue down to the atmospheric cut-off around $3200 \AA$, and it is normally not used longward of $8000 \AA$ for the fringing that affects the sensor. The long-slit spectra were recorded with a $300 \mathrm{ln} / \mathrm{mm}$ grating blazed at $5000 \AA$, and extend spatially for 9.5 arcmin (at a scale of $1.119 \mathrm{arcsec} / \mathrm{pix}$ ) covering the wavelength range from 3300 to $8000 \AA$ at $2.31 \AA /$ pix. The spectra were reduced within IRAF, carefully involving all steps connected with correction for bias, 
U. Munari et al.: A flash-ionized nebula around PNV J03093063+2638031

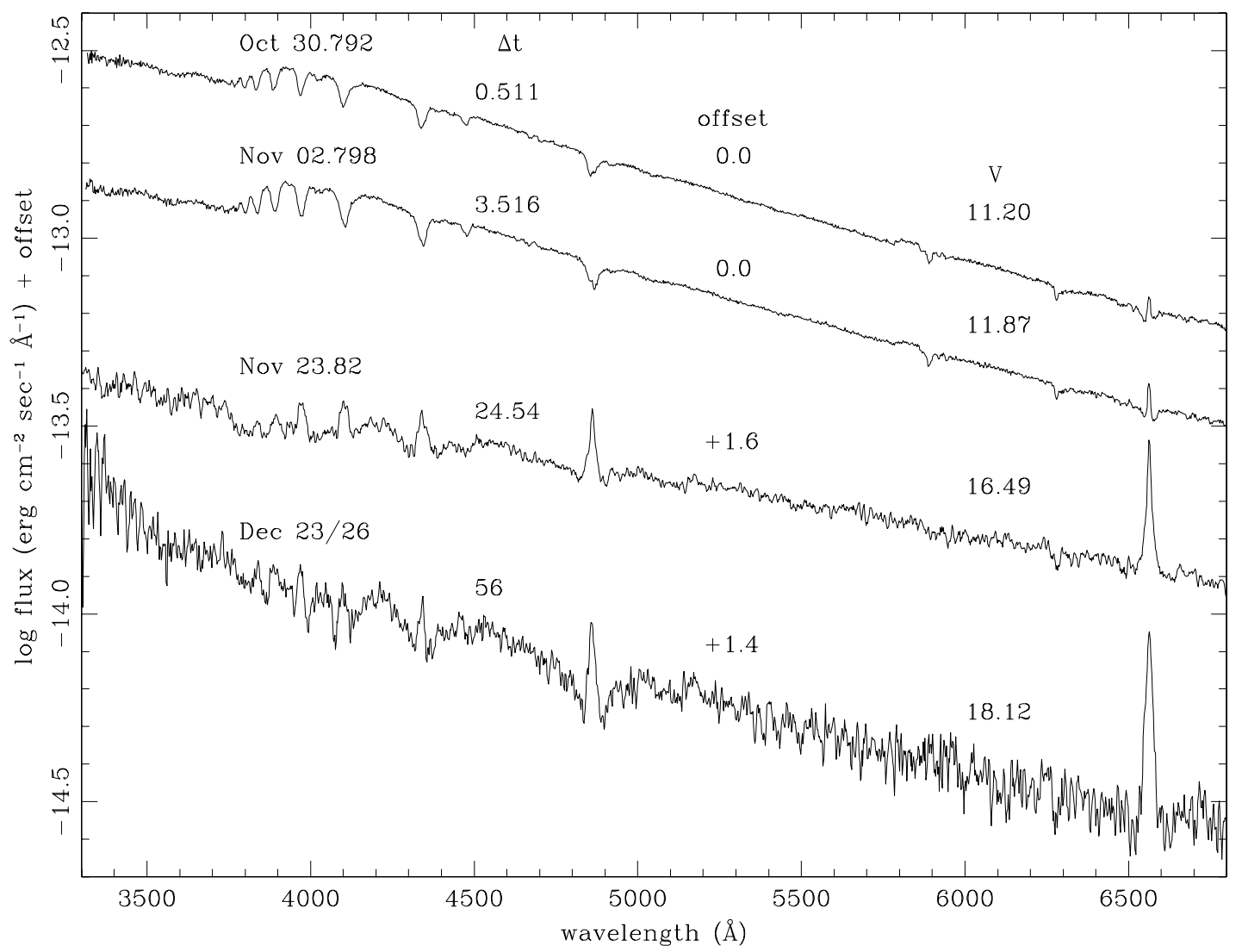

Fig. 2. Fluxed spectra of PNV J03093063+2638031 as observed with the Asiago $1.22 \mathrm{~m}+\mathrm{B} \& \mathrm{C}$ telescope. Note the different slope of Nov. 23.824 spectrum taken at the time of the reddest color displayed by PNV J03093063+2638031 (cf. Fig. 1).

Table 1. Our $B V R_{\mathrm{C}} I_{\mathrm{C}}$ photometry of PNV J03093063+2638031 during the 2014 superoutburst.

\begin{tabular}{cccccccccc}
\hline \hline $\begin{array}{c}\text { HJD } \\
(-2450000)\end{array}$ & $B$ & \pm & $V$ & \pm & $R_{\mathrm{C}}$ & \pm & $I_{\mathrm{C}}$ & \pm & $\mathrm{id}$ \\
\hline 6961.511 & 11.105 & 0.004 & 11.228 & 0.003 & 11.235 & 0.003 & 11.242 & 0.006 & $\mathrm{a}$ \\
6962.514 & 11.397 & 0.003 & 11.492 & 0.003 & 11.527 & 0.003 & 11.495 & 0.007 & $\mathrm{a}$ \\
6963.528 & 11.566 & 0.004 & 11.688 & 0.005 & 11.698 & 0.003 & 11.668 & 0.006 & $\mathrm{a}$ \\
6964.503 & 11.845 & 0.004 & 11.889 & 0.005 & 11.886 & 0.003 & 11.867 & 0.009 & $\mathrm{a}$ \\
6964.530 & 11.851 & 0.005 & 11.878 & 0.010 & 11.889 & 0.005 & 11.850 & 0.008 & $\mathrm{a}$ \\
6967.512 & 12.304 & 0.006 & 12.341 & 0.009 & 12.364 & 0.006 & 12.411 & 0.009 & $\mathrm{a}$ \\
6971.499 & 12.789 & 0.005 & 12.803 & 0.010 & 12.866 & 0.006 & 12.936 & 0.008 & $\mathrm{a}$ \\
6975.371 & 13.368 & 0.007 & 13.354 & 0.009 & 13.461 & 0.006 & 13.151 & 0.009 & $\mathrm{a}$ \\
6975.508 & 13.425 & 0.008 & 13.398 & 0.061 & 13.505 & 0.009 & 13.173 & 0.010 & $\mathrm{a}$ \\
6981.478 & 14.658 & 0.012 & 14.706 & 0.028 & 14.555 & 0.018 & 14.393 & 0.024 & $\mathrm{a}$ \\
6984.518 & 16.985 & 0.075 & 16.485 & 0.098 & 16.278 & 0.073 & 15.934 & 0.081 & $\mathrm{a}$ \\
6990.476 & 17.510 & 0.135 & 16.930 & 0.121 & & & & & $\mathrm{a}$ \\
7001.414 & 17.691 & 0.259 & 17.340 & 0.179 & & & & & $\mathrm{a}$ \\
7010.264 & 18.032 & 0.075 & 17.815 & 0.031 & 17.636 & 0.075 & 16.741 & 0.138 & $\mathrm{~b}$ \\
7018.307 & 18.052 & 0.025 & 18.121 & 0.015 & 18.095 & 0.045 & & & $\mathrm{c}$ \\
7026.227 & 18.064 & 0.071 & 18.096 & 0.085 & 17.851 & 0.097 & & & $\mathrm{~b}$ \\
7034.274 & 18.178 & 0.015 & 18.170 & 0.039 & 18.040 & 0.015 & & & $\mathrm{c}$ \\
7065.232 & 18.412 & 0.084 & 18.378 & 0.119 & 18.146 & 0.071 & & & $\mathrm{~b}$ \\
7095.363 & 18.347 & 0.073 & 18.301 & 0.063 & & & & & $\mathrm{c}$ \\
\hline
\end{tabular}

Notes. The last column identifies the telescopes: $a=$ ANS 011, $b=$ Asiago 67/92 cm Schmidt, $c=$ Asiago $1.82 \mathrm{~m}+$ AFOSC.

dark and flat, sky subtraction, wavelength, and flux calibration. The error on the flux calibration at all epochs should not exceed $6 \%$ anywhere, as the intercalibration of the spectrophotometric standards suggests, and the comparison with the nearsimultaneous $B V R_{\mathrm{C}} I_{\mathrm{C}}$ confirms. Table 2 provides a log-book of the spectroscopic observations and the spectra of PNV at the first four epochs are plotted in Fig. 2.
Table 2. Logbook of our spectroscopic observations of PNV J03093063+2638031 during the 2014 superoutburst.

\begin{tabular}{rccccc}
\hline \hline Date & $\langle$ UT $\rangle$ & $\begin{array}{c}\text { JD } \\
(-2450000)\end{array}$ & $\begin{array}{c}\Delta t \\
(\text { days })\end{array}$ & $\begin{array}{c}\text { Expt } \\
(\mathrm{s})\end{array}$ & $\begin{array}{c}\text { Slit } \\
\text { orient }\end{array}$ \\
\hline 20141030 & $19: 02$ & 6961.293 & 0.511 & 600 & $\mathrm{E}-\mathrm{W}$ \\
1102 & $19: 09$ & 6964.298 & 3.516 & 720 & $\mathrm{E}-\mathrm{W}$ \\
1123 & $19: 38$ & 6985.318 & 24.54 & 3600 & $\mathrm{E}-\mathrm{W}$ \\
1223 & $19: 37$ & 7015.317 & 54.54 & 5400 & $\mathrm{E}-\mathrm{W}$ \\
1223 & $21: 56$ & 7015.414 & 54.63 & 5400 & $\mathrm{~N}-\mathrm{S}$ \\
1226 & $19: 54$ & 7018.329 & 57.55 & 5400 & $\mathrm{E}-\mathrm{W}$ \\
1226 & $23: 30$ & 7018.479 & 57.70 & 5400 & $\mathrm{~N}-\mathrm{S}$ \\
20150311 & $19: 09$ & 7093.293 & 132.5 & 7200 & $\mathrm{E}-\mathrm{W}$ \\
0312 & $19: 24$ & 7094.308 & 133.5 & 3600 & $\mathrm{E}-\mathrm{W}$ \\
\hline
\end{tabular}

Notes. All spectra have been obtained with the Asiago $1.22 \mathrm{~m}+\mathrm{B}$ and $\mathrm{C}$ telescope, at a dispersion of $2.31 \AA /$ pix and covering the range $3300-7950 \AA . \Delta t$ is counted from optical maximum.

\section{Progenitor}

At the improved position for PNV derived in Sect. 1, a faint star is clearly visible on both Palomar I and II sky survey images, which was also detected by various surveys and listed in several catalogs at the positions given in Table 3 . The straight average of their end figures is $29.744( \pm 0.020)$ and $04^{\prime \prime} .39( \pm 0.10)$, coincident within combined errors with the observed position of PNV.

The PNV progenitor is a faint and blue star. Its USNOB1.0 magnitudes on Palomar I and II surveys are $B 1=19.18$, $R 1=18.66$ and $B 2=19.22, R 2=18.14, I 2=18.70$. The GSC 2.3 catalog reports for PNV $B=18.80, V=18.25$, and $I=18.35$. The SDSS survey measured PNV progenitor 
Table 3. Cross-identification of PNV J03093063+2638031 with other catalogs and the respective astrometric positions.

\begin{tabular}{llcc}
\hline \hline Catalog & Identification & $R A(J 2000)$ & Dec (J2000) \\
\hline USNO-B1.0 & $1166-0037733$ & 030929.745 & +263804.66 \\
USNO-A2.0 & $1125-01018475$ & 030929.638 & +263804.49 \\
SDSS-ID & $7781-301-2-0229-0414$ & 030929.771 & +263804.25 \\
ALLWISE & J030929.78+263804.1 & 030929.789 & +263804.20 \\
PPMXL & 3037362365462015717 & 030929.755 & +263804.45 \\
GSC2.3 & NCGZ009444 & 030929.730 & +263804.01 \\
GALEX & 6373202824497465728 & 030929.783 & +263804.70 \\
\hline
\end{tabular}

at $u^{\prime}=19.137 \pm 0.031, g^{\prime}=18.882 \pm 0.009, r^{\prime}=18.907 \pm$ $0.012, i^{\prime}=19.011 \pm 0.017$ and $z^{\prime}=18.969 \pm 0.050 \mathrm{mag}$. It was also detected by the GALEX ultraviolet survey at FUV = $19.606 \pm 0.210$ and NUV $=19.607 \pm 0.128 \mathrm{mag}\left(\lambda_{\mathrm{FUV}}=1540 \AA\right.$ and $\lambda_{\mathrm{NUV}}=2315 \AA$ ). The object was too faint for detection by 2 MASS at $J, H$ and $K_{\mathrm{s}}$ wavelengths, but it was detected at mid-infrared wavelengths by AllWISE survey at magnitudes $W 1=16.397 \pm 0.082(S / N=13.3)$ and $W 2=16.311 \pm 0.275$ $(S / N=4.0)$, where $\lambda_{W 1}=3.35 \mu \mathrm{m}$ and $\lambda_{W 2}=4.6 \mu \mathrm{m}$. The AllWISE program (Cutri et al. 2013, VizieR On-line Data Catalog II/328) extends the work of the WISE space mission (Wright et al. 2010) by combining data from the cryogenic and post-cryogenic survey phases.

The spectral energy distribution of PNV progenitor is presented in Fig. 3. It is well fitted by a $F_{\lambda} \propto \lambda^{-2}$ power law over the whole UV-opt-IR wavelength range covered by GALEX, SDSS, and AllWISE observations, and is compatible with an accretion disk dominating the emission from the system in quiescence (La Dous 1989). The completeness limits of 2MASS survey in the area around PNV are $J \sim 16.5, H \sim 16.0$, and $K \sim 15.5$ and the corresponding fluxes lie well above the fitting line in Fig. 4, in agreement with the nondetection of PNV by 2MASS. For reference purposes it is useful to list the conventional Johnson-Cousins and 2MASS magnitudes corresponding to the linear fit in Fig. 3: $U=18.76, B=19.51, V=19.53$, $R_{\mathrm{C}}=19.23, I_{\mathrm{C}}=19.09, J=18.58, H=18.11$, and $K_{\mathrm{s}}=17.55$.

\section{Historical light curve from Harvard plates}

The superoutbursts in WZ Sge stars are separated by decades, and the normal outbursts in between are rare or even absent. To test if this is also the case for PNV, we went to the Harvard plate stack in Cambridge (USA) to inspect historical photographs covering the position of PNV. The vast majority of the plates at Harvard are blue sensitive and exposed through lenseastrographs without intervening photometric filters. In modern terms, this is roughly equivalent to observing in the $B$-band. The 2014 superoutburst peaked at $B \sim 10.8$, so we narrowed our search to plates going deep enough to reach at least a limiting magnitude of $\sim 10.8$ in blue light. Out of the $\sim 600$ plates that we inspected, 332 of them were of a photometric quality meeting such criterion, covering the time interval from Jan. 1899 to Nov. 1981. Given the short focal length of most of the astrographs used to expose the Harvard plates, we augmented the photometric sequence around PNV (described in Sect. 2 and optimized for the longer focal length CCD observations obtained during the 2014 superoutburst) with additional stars spanning a wider range in magnitude and at greater angular distances from PNV. They too were selected from the APASS survey. The final sequence extends from $B=10.152$ to $17.155 \mathrm{mag}$, and it was used at the microscope eyepiece to estimate the brightness of

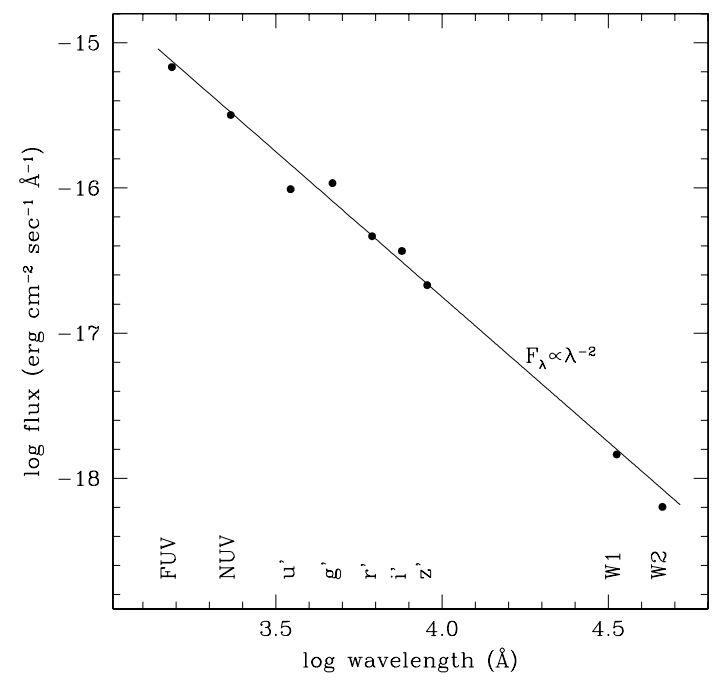

Fig. 3. Spectral energy distribution of PNV J03093063+2638031 in quiescence, combining nonsimultaneous observations by GALEX, SDSS, and AllWISE.

PNV or the limiting magnitude of the plate, which is defined as the midpoint magnitude between the faintest sequence star still visible and the brightest of those not visible.

The limiting magnitude of the 332 plates ranged from 10.8 to 16.5 , and PNV was too faint to be detected on all of them. The results are listed in Table 4 and are plotted in Fig. 4. During the 2014 superoutburst, PNV took less than a month to decline below the limiting magnitude of the deepest Harvard plates. Such a fast evolution could easily accommodate similar events in the time gaps uncovered by the Harvard plates, including those due to solar conjunction. Nonetheless, the very fact that PNV went undetected on all 332 plates we inspected suggests that its outbursts are a rare occurrence, in nice agreement with the wellestablished behavior of WZ Sge stars as a class.

\section{The 2014 outburst}

The photometric evolution of the outburst is presented in Fig. 1. It is similar to that seen in the other WZ Sge-type objects (e.g. Nogami et al. 2009; Chochol et al. 2012), and is composed of three distinct parts.

We began our observations on JD $=6961.511$, about one day past the original discovery observations by S. Ueda, and as soon as darkness allowed after the announcement of the discovery was posted to TOCP. We caught the object already declining from maximum, $\sim 0.2 \mathrm{mag}$ below it. S. Kiyota (Japan, as reported on TOCP) obtained $B=10.83, V=11.02$ and $I_{\mathrm{C}}=10.92$ on Oct. 30.28 UT (JD $=2456960.78)$, which we take to mark the optical maximum and that is plotted as an open circle in Fig. 1. According to the discovery report by S. Ueda, nothing was visible down to $13 \mathrm{mag}$ at the position of PNV two days before the discovery (marked by the arrow in Fig. 1). Therefore, the rise to maximum was (very) fast and, upon hitting maximum brightness, PNV immediately began declining. The initial decline, usually referred to as the "plateau" in literature, developed at a nearly constant $B-V \sim 0.0$ and took PNV from peak $V=11.0$ down to $V=14.0$ in 19 days. The spectra of PNV soon after maximum (cf. Fig. 2) are typical of CV variables in outburst, with a strong A-type continuum and broad hydrogen Balmer and HeI absorption lines. The Balmer continuum 


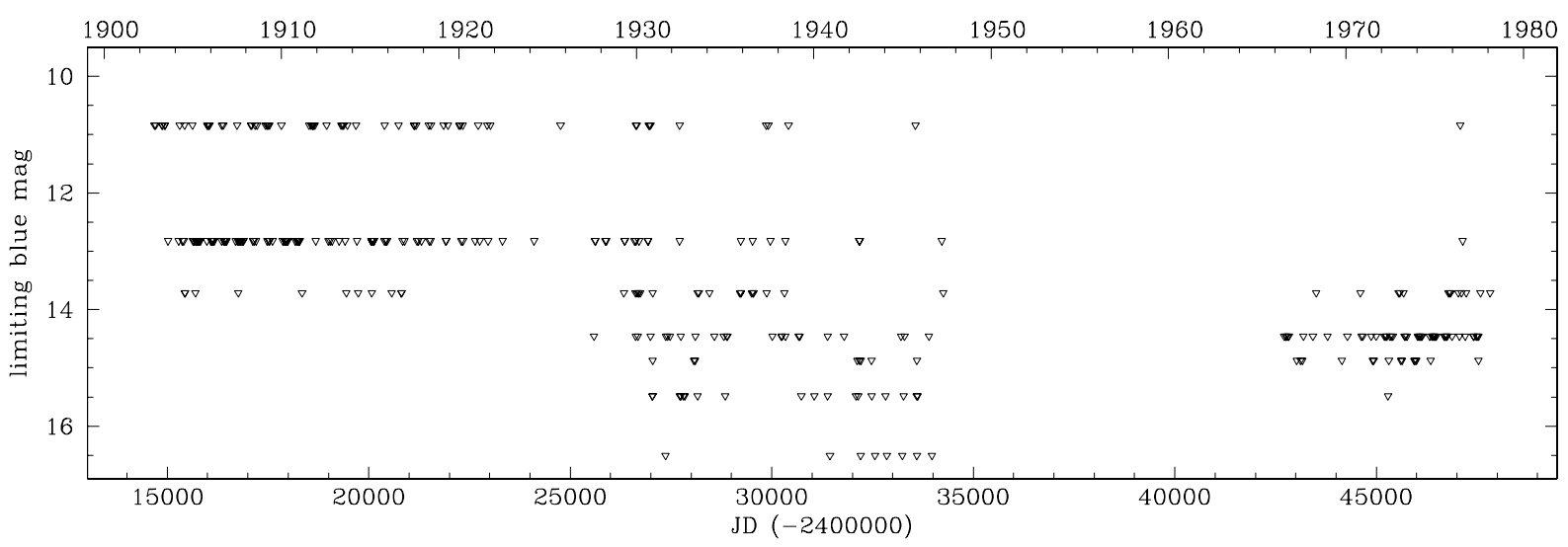

Fig. 4. Distribution in time of the "fainter than" limits to the B magnitude of PNV J03093063+2638031 on 332 plates of the Harvard plate stack, which we inspected looking for previous outbursts.

is also in absorption and a weak emission core is visible in $\mathrm{H} \alpha$ and marginally in $\mathrm{H} \beta$.

This first phase was followed by a very rapid drop in brightness, from $V=14.0$ to $V=16.5$ in just four days, which was accompanied by cooling of the $B-V$ color from 0.0 to +0.6 mag. This change in color is nicely confirmed by the change in the slope of the continuum of the corresponding spectrum shown in Fig. 2. Following this rapid drop, the spectrum of PNV developed strong hydrogen Balmer emission lines, the absorption in the Balmer continuum vanished and early hints emerged of the very wide hydrogen Balmer absorption lines from the underlying white dwarf. The apparent reinforcing of the Balmer emission lines was actually driven by the rapid drop in intensity of the underlying continuum. In fact, the integrated flux of the $\mathrm{H} \alpha$ emission line for the four spectra presented in Fig. 2 declined steadily with time: it was $105,81,9.8$ and $5.9 \times 10^{-15} \mathrm{erg} \mathrm{cm}^{-2} \mathrm{~s}^{-1}$, respectively.

The third and last phase saw PNV slowly complete the final decline toward quiescence brightness, in pace with a gradual warming of the $B-V$ color from +0.6 back to $\sim 0.0$ mag. This phase took PNV from $V=16.5$ to $V=18.1$ in $\sim 35$ days, and to $V=18.4$ in an additional $\sim 45$ days. This is still one mag brighter than the quiescence level, taken to be represented by the linear fit of Fig. 3 (see Sect. 3), suggesting that the return to proper quiescence required significant additional time. Our spectrum of PNV obtained when its was $V=18.1 \mathrm{mag}$, displays strong and very wide Balmer absorption lines from the underlying white dwarf. $\mathrm{H} \alpha$ emission completely fills in the corresponding absorption line, while higher Balmer lines are reduced to just emission cores. This is the combined effect of a reduction in the integrated flux of emission lines for higher term Balmer lines $\left(5.9,3.7\right.$, and $1.9 \times 10^{-15} \mathrm{erg} \mathrm{cm}^{-2} \mathrm{~s}^{-1}$ for $\mathrm{H} \alpha, \mathrm{H} \beta$, and $\mathrm{H} \gamma$, respectively) and the underlying white dwarf spectrum rapidly increasing in intensity toward the blue. No terminal echo outbursts, of the type displayed, for example, by EG Cnc (Patterson et al. 1998), was observed in PNV during the return to quiescence brightness (cf Fig. 1).

\section{Discovery of a large, spatially resolved and outburst-ionized emission nebula around PNV J03093063+2638031}

A very peculiar, probably unique feature of PNV among known WZ Sge-type stars, is the detection of a spatially extended nebula centered on the variable, which became briefly visible because
Table 4. Upper limits to the magnitude of PNV J03093063+2638031 on the 332 Harvard plates we inspected looking for previous unrecorded outbursts.

\begin{tabular}{cccccc}
\hline \hline JD & Date & UT & $\begin{array}{c}\text { Plate } \\
\text { series }\end{array}$ & $\begin{array}{c}\text { Plate } \\
\text { N. }\end{array}$ & $\begin{array}{c}\text { Lim. } \\
\text { mag }\end{array}$ \\
\hline 2427472.54 & $1934-02-04$ & $01: 02$ & rh & 5714 & 14.5 \\
2427712.75 & $1934-10-02$ & $05: 52$ & rh & 6212 & 10.8 \\
2427712.80 & $1934-10-02$ & $07: 01$ & ac & 32361 & 12.8 \\
2427718.86 & $1934-10-08$ & $08: 30$ & rh & 6236 & 15.5 \\
2427740.70 & $1934-10-30$ & $04: 45$ & ac & 32422 & 14.5 \\
2427741.73 & $1934-10-31$ & $05: 16$ & rh & 6287 & 15.5 \\
2427808.55 & $1935-01-06$ & $01: 11$ & bm & 335 & 15.5 \\
2427840.56 & $1935-02-07$ & $01: 23$ & rh & 6442 & 15.5 \\
2428067.86 & $1935-09-22$ & $08: 32$ & ac & 32837 & 14.9 \\
2428095.72 & $1935-10-20$ & $05: 02$ & ac & 32895 & 14.9 \\
2428104.75 & $1935-10-29$ & $05: 48$ & ca & 535 & 14.5 \\
2428156.52 & $1935-12-20$ & $00: 24$ & ac & 32967 & 13.7 \\
2428159.58 & $1935-12-23$ & $01: 47$ & rh & 6941 & 15.5 \\
\hline
\end{tabular}

Notes. The table is published in its entirety at the CDS. A portion is shown here for guidance regarding its form and content.

it was ionized by the 2014 outburst, proving its firm physical association to PNV. We discovered it during the spectroscopic observations with the Asiago $1.22 \mathrm{~m}$ telescope, carried out in long-slit mode with the slit height extending for about 9.5 arcmin on the sky. The nebula is not of the reflection type (thus producing a light-echo of the outburst) because it is only visible in the Balmer emission lines and not in the continuum (which was brighter than the emission lines around outburst maximum).

On the first observation on 30 Oct. 2014 (day +0.51) there is no hint of spectral emission features spatially extending more than the stellar continuum. However, on the next spectrum obtained on 2 Nov., day +3.52 , the $\mathrm{H} \alpha$ emission was already noticeably extended in a similar manner on both sides of the stellar spectrum. The spatial FWHM of the stellar spectrum (dominated by seeing dispersion) was 3.92 pixels, and that of the $\mathrm{H} \alpha$ was 11.62 pixels. This corresponds to a seeing-corrected $F W H M(\mathrm{H} \alpha)=12.2 \operatorname{arcsec}$.

From this we may estimate a distance to PNV if we assume that this extended $\mathrm{H} \alpha$ emission is produced within a spherical and homogeneous nebula centered on PNV, which begins to emit by recombination as soon as the ionizing radiation from the outbursting central star reaches it. The time passed from discovery (when the object was already close to maximum brightness) to our spectroscopic detection of the extended 


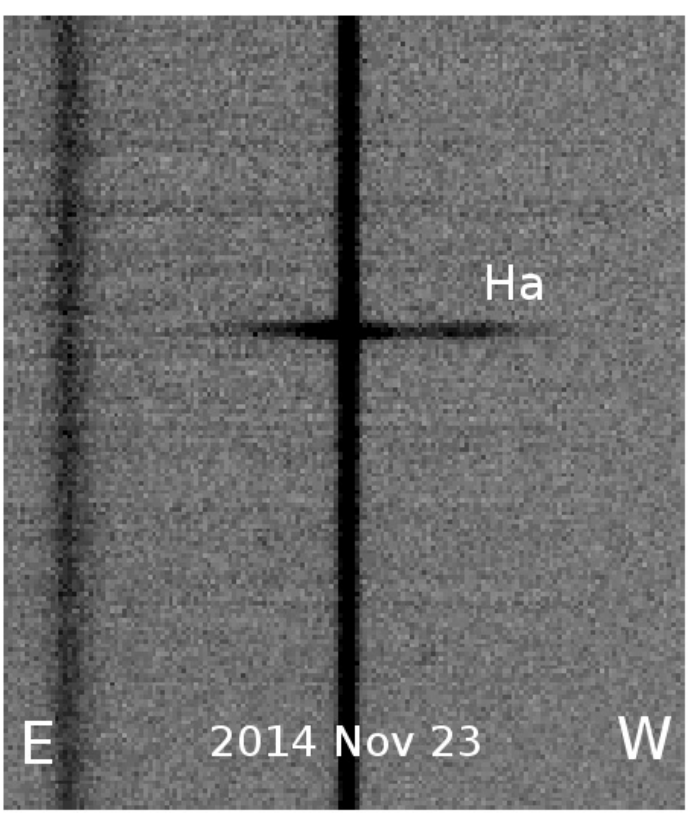

distance in arcsec

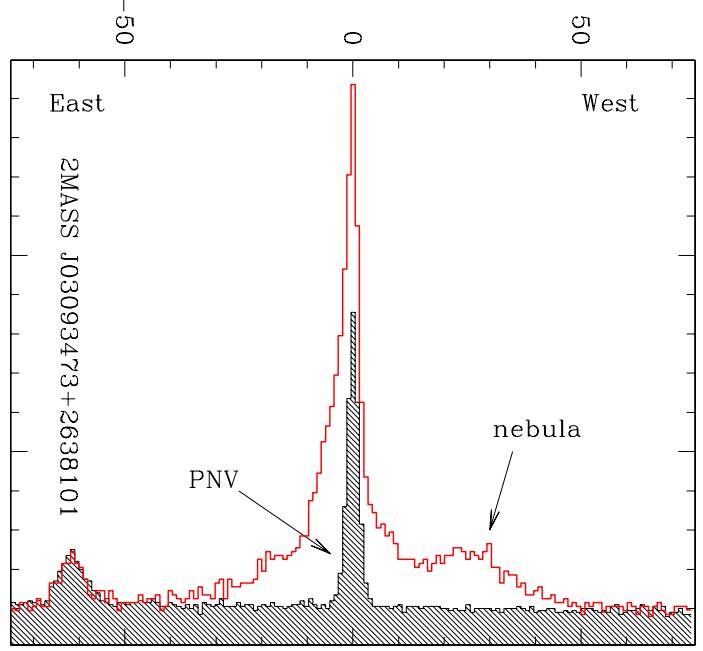

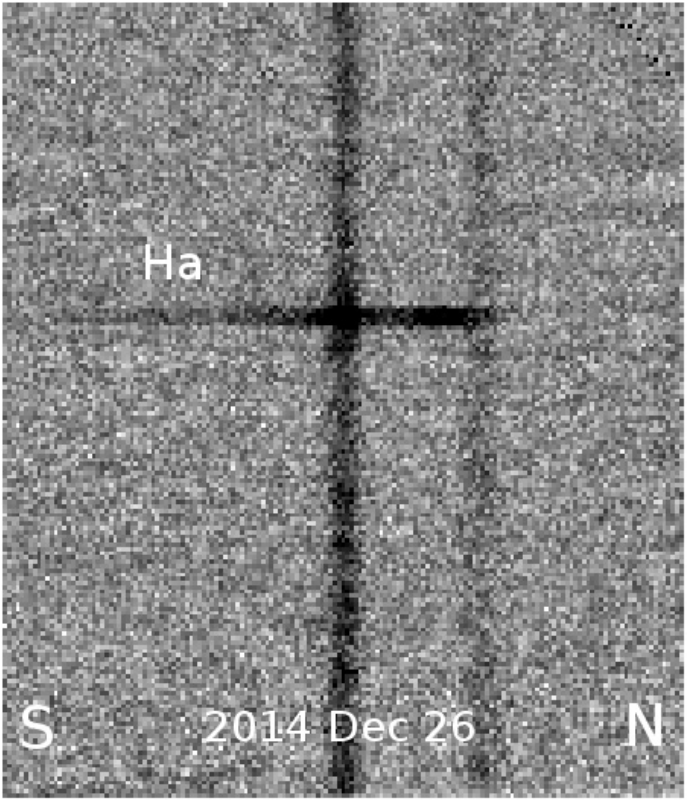

distance in arcsec

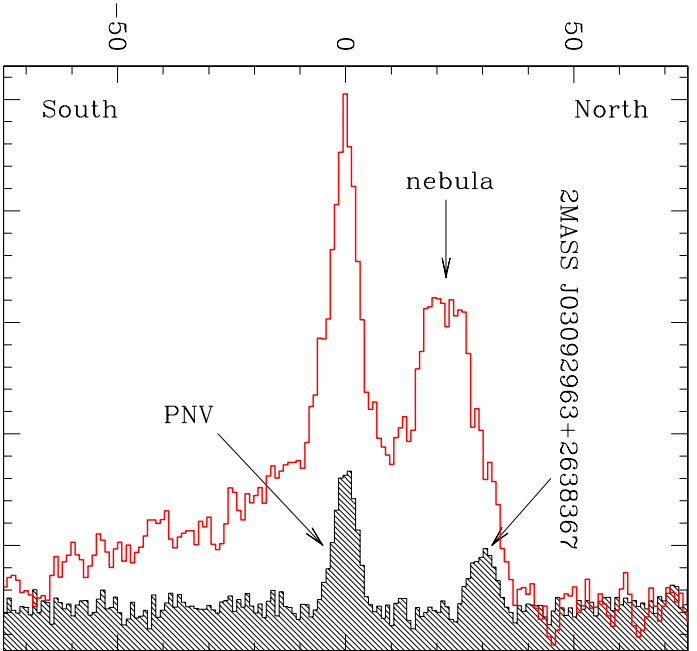

Fig. 5. Top left: part of the 2D low-resolution spectrum of PNV J03093063+2638031 we obtained on 23 Nov. 2014, when the object was shining at $V=16.7$ during the advanced decline from outburst maximum. The wavelength range covers from $6393 \AA$ at the top to $6821 \AA$ at the bottom and shows the $\mathrm{H} \alpha$ spatially extended nebula as recorded with the spectrograph slit alligned east-west. The weak and fuzzy spectrum at left is that of 2MASS 03093473+2638101, a nearby $R=12.4$ mag star barely intercepted by the slit. Bottom left: tracing along the spatial axis of the same spectrum of PNV J03093063+2638031 shown on the top panel. The thicker red line traces along the emission from the nebula, while the shaded profile traces just away from the nebula to provide a reference. Right panels: the same for the 26 Dec. 2014 spectrum, this time with the slit oriented north-south and when PNV J03093063+2638031 was at $V=18.1 \mathrm{mag}$. The weak spectrum at right is that of 2MASS 03092963+2638367, a nearby $R=17.5$ mag star marginally intercepted by the slit.

nebula was 4.17 days. Assuming that the rise to maximum of PNV was nearly instantaneous, this means that the outer radius of the ionized volume was at that time $1.1 \times 10^{16} \mathrm{~cm}$ from PNV, or $3.5 \times 10^{-3} \mathrm{pc}$. If we compare this radius with the FWHM of 12.2 arcsec, the distance turns out to be $\sim 120 \mathrm{pc}$, and the absolute magnitude in quiescence is $M_{V}=14.1$ (assuming a negligible extinction at the high galactic latitude of PNV).

The next time we observed PNV was 21 days later (Nov. 23) when the star was $\sim 4.6$ mag fainter. The bright plateau past maximum was over by $\sim 6$ days and with it the sustained input of ionizing photons to the circumstellar medium. By this time the spatial extent of the ionized nebula had significally grown. The appearance of the spatially extended $\mathrm{H} \alpha$ emission on this spectrum is shown in the left panel of Fig. 4. The outer portion of the nebula extends to 40 arcsec toward the east and 50 arcsec to the west of PNV, with considerable substructures visible in the intensity profile, indicating large departures from spherical symmetry in the distribution of the gas.

A month later, we invested considerable observing time at two distinct and nearby dates (Dec. 23 and 26) to obtain longslit spectra of PNV at two perpendicular orientations, east-west and north-south. The spatially extended $\mathrm{H} \alpha$ emission along the east-west was similar to that of a month earlier, only with a reduced brightness. This indicates that not much gas exists external to that already ionized by Nov. 23 (density bounded nebula), and that the ionized gas was already completing the recombination given the long gone photoionization from the central star. The spatially extended profile along the north-south direction for Dec. 26 observations is shown in the right panel of Fig. 4. The $\mathrm{H} \alpha$ profile along the north-south direction extends by similar 
amounts compared to east-west: 35 arcsec toward north and 65 arcsec toward south, indicating that PNV sits approximately at the projected center of the nebula. Considerable substructures are visible also along the north-south spatial extention of the nebula, as it is for the east-west.

To check on the photoionization/recombinations scenario for the nebula, we reobserved PNV with the same instrumentation in March 2015, exposing much longer than previous visits. No trace of the nebula was visible, indicating that recombination within the ionized gas was by that time completed and the circumstellar nebula had returned to the obscurity from which it had briefly emerged during the superoutburst of 2014. The great surface brightness it displayed (expecially at the time we discovered it) would have resulted in an easy detection on the red Palomar Sky Survey plates, but they shows just empty sky at the position of the nebula.

\section{Discussion}

At the limited spectral resolution of our spectra, the spectral width of the spatially extended $\mathrm{H} \alpha$ is comparable to that of the lines from the NeArFe calibration lamp or the night-sky lines (their FWHM corresponds to $\sim 250 \mathrm{~km} \mathrm{~s}^{-1}$ at $\mathrm{H} \alpha$ wavelengths). This means that the local velocity dispersion within the circumstellar gas is low, in particular the gas is not expanding quickly. The nebula also does not rotate with a large angular velocity, nor it is subject to large scale motions because its emission is centered at the same wavelength along its whole spatial extent. In short, the circumstellar nebula looks like a quiet blob of gas, with considerable substructures and a clear physical association to PNV.

The hydrogen recombination timescale (in hours) is related to electron temperature and density by

$t_{\mathrm{rec}}=1.15\left(\frac{T_{\mathrm{e}}}{10^{4} \mathrm{~K}}\right)^{0.8}\left(\frac{n_{\mathrm{e}}}{10^{9} \mathrm{~cm}^{-3}}\right)^{-1}$

following Ferland (1997). With an observed recombination timescale of the order of $\sim$ two months, the electron density is $4.5,8$ or $14 \times 10^{5} \mathrm{~cm}^{-3}$ for $T_{\mathrm{e}}=5000,10000$ or $20000 \mathrm{~K}$, respectively.

With a mean angular extension of 50 arcsec and a distance of $120 \mathrm{pc}$, the physical radius of the nebula is $0.03 \mathrm{pc}$, or $1 / 10$ of a typical planetary nebula. If homogeneously filled by hydrogen at the density estimated above for $T_{\mathrm{e}}=10000 \mathrm{~K}$, its mass would be $2 M_{\odot}$. The substructures well visible in Fig. 4 suggest that a sizeable fraction of the available volume is indeed empty. Even if the filling factor was just a tiny $1 \%$, still the amount of mass in the nebula would be orders of magnitude greater than that ejected by outbursts on white dwarfs, such as nova eruptions that, depending on the mass of the white dwarf, are observed to expel from $10^{-6}$ to $10^{-4} M_{\odot}$ (Bode \& Evans 2008). Furthermore, the ejection velocities observed in novae (from several hundreds to a few thousand $\mathrm{km} \mathrm{s}^{-1}$, Warner 1995) are much larger than the low (if any) expansion velocity observed for the nebula around PNV.

Appreciable mass ejection cannot be expected from WZ Sge-type binaries based on their nature. These systems are composed of a white dwarf orbited every $\sim 80 \mathrm{~min}$ by a brown dwarf, the mass of the latter being of the order of a mere $\sim 0.06 M_{\odot}$ (Hellier 2001), which is below the limit for stable hydrogen burning in the core. The mass transfer rate in this kind of system is extremely low, $\sim 10^{-12} M_{\odot} / \mathrm{yr}$, and unable to sustain the presence of the nebula. If all the material transferred from the brown dwarf to the white dwarf eventually ended up feeding the circumstellar nebula, it would take more than the age of the Universe to accumulate the nebula mass corresponding to a tiny $1 \%$ filling factor $\left(0.02 M_{\odot}\right)$. In addition, a filling factor larger that a few percent would make the nebula mass greater than the mass of the brown dwarf itself. Thus, it is highly improbable that the nebula around PNV originated from the brown dwarf companion.

One could speculate that the nebula around PNV is the remnant of the slow wind the AGB progenitor blown off before turning into the present day white dwarf. There are however several problems with this origin also. The dilution of AGB wind into the surrounding instertellar space is rapid, of the order of $10^{4} \mathrm{yr}$, which is the age of common planetary nebulae, and the evolutionary path leading to the formation of WZ Sge-type binaries is instead much longer than that $\left(\sim 10^{9} \mathrm{yr}\right)$. Furthermore, AGB winds produce a lot of dust. In fact, planetary nebulae show clear signs of thermal emission from dust mixed with the gas, but the 2MASS and AllWISE data indicate that no detectable dust is present in PNV.

The circumstellar nebula that should not exist is instead stubbornly there, with a clear physical association to PNV as proven by the sudden photoionization induced by the 2014 superoutburst. In light of the challenges this nebula seems to pose to theoretical models, it is highly advisable to search for similar nebulae around future WZ Sge-type transients. This will require spectrographs equipped with long slits and the investment of long integration times, which is not easy to accommodate in present times dominated by remote service observing and multiobject, fiber-fed spectrographs.

Acknowledgements. We would like to thank Alison Doane, Curator of Astronomical Photographs at the Harvard College Observatory, for granting access to the Harvard plate stack, and Stella Kafka for hospitality at AAVSO Headquarters. This work was supported in part by the University of Rijeka under the project number 13.12.1.3.03. and by the Croatian Science Foundation under the project 6212 Solar and Stellar Variability.

\section{References}

Bode, M. F., \& Evans, A. 2008, Classical Novae (Cambridge University Press), 43

Chochol, D., Katysheva, N. A., Shugarov, S. Y., Zemko, P. O., \& Andreev, M. V. 2012, CoSka, 42, 39

Ferland, G. J. 1997, An Introduction to Cloudy, University of Kentucky, Lexington

Hellier, A. 2001, Cataclysmic Variable Stars (Chichester, UK: Springer \& Praxing Publishing)

Henden, A., \& Munari, U. 2014, CoSka, 43, 518

Henden, A. A., Levine, S. E., Terrell, D., Smith, T. C., \& Welch, D. 2012, JAVSO, 40, 430

Kuulkers, E., Henden, A. A., Honeycutt, R. K., et al. 2011, A\&A, 528, A152

La Dous, C. 1989, A\&A, 211, 131

Munari, U., \& Moretti, S. 2012, Balt. Astron., 21, 22

Munari, U., Bacci, S., Baldinelli, L., et, al. 2012, Balt. Astron., 21, 13

Munari, U., Henden, A., Frigo, A., et al. 2014, AJ, 148, 81

Nogami, D., Hiroi, K., Suzuki, Y., et al. 2009, ASP Conf. Ser., 404, 52

Patterson, J., Richman, H., Kemp, J., \& Mukai, K. 1998, PASP, 110, 403

Santangelo, M., M. M., \& Gambogi, S. 2014, ATel, 6653, 1

Warner, B. 1995, Cataclysmic variable stars, Camb. Astrophys. Ser., 28

Wright, E. L., Eisenhardt, P. R. M., Mainzer, A. K., et al. 2010, AJ, 140, 1868 